\title{
Negatively Charged Donors in Flat Quantum Dots
}

\author{
Luis F. García*, Jairo H. Marín ${ }^{\dagger}$, and Ilia D. Mikhailov* \\ *Universidad Industrial de Santander. A.A.678, Bucaramanga, Colombia \\ $\dagger$ Universidad Nacional de Colombia A.A 3840. Medellín, Colombia
}

Received on 8 December, 2005

\begin{abstract}
The ground state energies of off-axis negatively charged donors in axially symmetrical quantum dots, with different shapes but in all cases with a small height-to-base radius aspect ratio are calculated in adiabatic approximation by using the Hylleraas-type trial function. The dependencies of the neutral and negative donor binding energies and their ratios on the base radius in the pyramid, lens and disk are calculated and compared with previously obtained results for the spherical quantum dot. We also present the contour plots of the binding energies of the neutral and negative donors with different positions along a vertical cross section in the middle of the quantum dots.
\end{abstract}

Keywords: Self-assembled quantum dot; Adiabatic approximation; Binding energies

\section{INTRODUCTION}

It is increasing the interest in the study of the electronic structure and properties of excitons, neutral, $\mathrm{D}^{0}$ and negatively charged, $\mathrm{D}^{-}$donors in the self-assembled quantum dots (SAQDs) which are formed by the Stranski-Krastanow growth mode by deposition a material on the substrate with different lattice parameter [1]. The electrical and optical properties of these structures may be changed in a controlled form by doping the shallow impurities whose energy levels are defined by the interplay between the reduction of the physical dimension, the Coulomb attraction and the inter-particle correlation. Previously, it has been shown that the electronic spectrum of $\mathrm{D}^{0}$ and $\mathrm{D}^{-}$in a spherical quantum $\operatorname{dot}(\mathrm{QD})$ is very sensible to the variation of the heterostructure size and the donor position [2]. In particular, it was established that the confinement leads to the enhancement of the binding energies both $E_{b}\left(D^{0}\right)$ of the neutral donor and $E_{b}\left(D^{-}\right)$of the negatively charged donor in such way that the ratio $\sigma=E_{b}\left(D^{-}\right) / E_{b}\left(D^{0}\right)$ successively increases with the decrease of the dimensionality of the structure. For example, this ratio for the on-center donor in a spherical QD (SQD) increases from 0.055 as the QD's radius is very large up to 0.3 as the QD's radius becomes less than the effective Bohr radius [3], i.e. the difference between binding energies within the heterostructures there is no longer as significant as in bulk. It would be interesting to extend this results obtained previously for a spherical quantum dot to SAQDs with different shapes.

The calculation of the energy spectrum of both the $\mathrm{D}^{0}$ and the $\mathrm{D}^{-}$confined in a heterostructure that has not a high symmetry as a QD requires a lot of computational efforts. To simplify these calculations in the papers [4] it has been used a simple variational procedure, which permits to reduce the wave equations for $\mathrm{D}^{0}$ and $\mathrm{D}^{-}$confined in semiconductor heterostructures to the similar equations for the hydrogen atom, $\mathrm{H}$ and negatively charged ion, $\mathrm{H}^{-}$in an isotropic effective space with a non-power-law dependence of the Jacobian on the electron-ion distance. It has been shown that the procedure gives for the $\mathrm{D}^{0}$ and $\mathrm{D}^{-}$in $\mathrm{QW}$, cylindrical QWW and spherical QD binding energies which are in an excellent concordance with the Monte Carlo and variational calculations [4].
The morphology of SAQDs generally is sufficiently complicated, and a direct calculation of the Jacobian similar to one for the SQD can not be fulfilled. But one can take an advantage of the adiabatic approximation (AA) applicable to these systems due to their small height-to-base radius aspect ratio. By using this approximation in this work we calculate the ground state energies of the neutral and negative donors in flat QDs with axial symmetry as a function of the distance from the donor position to the axis and the base radius of the quantum dot. Comparing results of calculation for structures with different geometry such as disk, lens and pyramid we analyze the QDs shape on donor's ground state energies.

\section{THEORETICAL MODEL}

In our calculation, we consider three different types of QDs with cylindrical symmetry whose 3D images are presented schematically in Fig.1: disk, lens and pyramid with the height $d_{0}$, the base radius $R_{0}$. Their profiles can be described by a functiond $(\rho)$, which defines the dependency of the thickness din $\mathrm{z}$-direction on the distance $\rho$ from the axis of symmetry. For the sake of the mathematical convenience we choose the dependencies $d(\rho)$ in a form of a piecewise continuous linear functions.

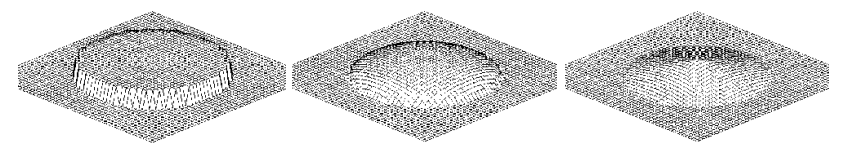

FIG. 1: The 3D images of QDs with different shapes: a) disk, b) lens, and c) pyramid.

The confinement potential due to the conduction band discontinuity in the junction of the QD is given by the piecewise constant function $V(\rho, z)$ which is equal to zero in the well $\left(0<z<d(\rho)\right.$ and equal to $V_{0}$ in the barrier. The effective Bohr radius $a_{0}^{*}=\varepsilon \hbar^{2} / m^{*} e^{2}$ and the effective Rydberg $R y^{*}=$ $e^{2} / 2 a_{0}^{*} \varepsilon$ are used in our calculations as units of length and energy, respectively. Differences between material parameters, 
the electron effective mass $m *$ and the dielectric constant $\varepsilon$, in the well and in the barrier are not included in the calculation. In what follows we present the results of calculation for the heterostructures of $\operatorname{In}_{0.55} \mathrm{Al}_{0.45} \mathrm{As} / \mathrm{Ga}_{0.65} \mathrm{Al}_{0.35} \mathrm{As}$ where the values of $a_{0}^{*}$ and $R y^{*}$ are equal to $8.86 \mathrm{~nm}$ and $6.40 \mathrm{meV}$, respectively, and the barrier height $V_{0} \approx 40 R y *$ [5]. It should be noted that in heterostructures of $\mathrm{GaAs} / \mathrm{Ga}_{0.7} \mathrm{Al}_{0.3}$ As where units of length and energy, $a_{0}^{*}$ and $R y^{*}$ are equal to $9.8 \mathrm{~nm}$ and $5.83 \mathrm{meV}$, respectively, the dimensionless barrier height have approximately the same value and therefore the results presented below are also applicable to these heterostructures.

For the potential $V(\rho, z)$ the one-particle Schrödinger equation do not completely separate and no exact solutions have been found. It is therefore some approximation method should be used to find the ground state wave function $f_{0}(\mathbf{r})$ of free electron corresponding to the lowest energy $E\left(e^{-}\right)$in a QD. In what follows we consider a typical situation when the QD's height is much smaller than its in-plane dimension $\left(d_{0}<<2 R_{0}\right)$, condition that allows us to take advantage of the AA. Following the AA standard scheme we represent $f_{0}(\mathbf{r})$ as a product of two wave functions, $f_{z}(z, \rho)$ which describes the rapid motion in the strongly confined transverse direction and $f_{\rho}(\rho)$ which corresponds to a slow inplane motion:

$$
f_{0}(\mathbf{r})=f_{z}(z, \rho) f_{\rho}(\rho)
$$

The function $f_{z}(z, \rho)$ and the associated lowest energy $E_{z}(\rho)$ can be found exactly as the well-known solution of the wave equation for a rectangular quantum well of width $d(\rho)$ and barrier height $V_{0}$ :

Once $f_{z}(z, \rho)$ and $E_{z}(\rho)$ are found, the function $f_{\rho}(\rho)$ may then be obtained as a solution of the two-dimensional central force problem with potential $E_{z}(\rho)$

$$
-f_{\rho}^{?}(\rho)-\frac{1}{\rho} f_{\rho}^{\prime}(\rho)+E_{z}(\rho) f_{\rho}(\rho)=E\left(e^{-}\right) f_{\rho}(\rho)
$$

To solve Eq. 2 we use the numerical procedure similar to trigonometric sweep method described in the paper [6]

To find the ground state wave functions $\Psi_{D^{o}}(\mathbf{r})$ for neutral donor and $\Psi_{D^{-}}\left(\mathbf{r}_{1}, \mathbf{r}_{2}\right)$ for a negatively charged donor in QD corresponding to the lowest energies $E\left(D^{o}\right)$ and $E\left(D^{-}\right)$, we use the following trial functions:

$$
\begin{aligned}
& \Psi_{D^{0}}(\mathbf{r})=f_{0}(\mathbf{r}) \Phi_{D^{0}}(|\mathbf{r}-\xi|) \\
& \Psi_{D^{-}}\left(\mathbf{r}_{1}, \mathbf{r}_{2}\right)=f_{0}\left(\mathbf{r}_{1}\right) f_{0}\left(\mathbf{r}_{2}\right) \Phi_{D^{-}}\left(\left|\mathbf{r}_{1}-\xi\right|,\left|\mathbf{r}_{2}-\xi\right|, r_{12}\right)
\end{aligned}
$$

Here $\xi$ and $\mathbf{r}_{i}(i=1,2)$ are vectors of the ion and electrons positions, respectively. The donor position in QDs with axial symmetry given by the vector $\xi$ below is described by two parameters, $\xi_{\rho}$, the distance from the axis and $\xi_{z}$, the distance from the base.

In Eq. 3, functions $\Phi_{D^{0}}$ and $\Phi_{D^{-}}$take into account the effect of the correlation produced by the Coulomb interaction. As it has been demonstrated in [4], the trial functions (3) satisfy the Schrödinger variational principle, if $\Phi_{D^{0}}$ and $\Phi_{D^{-}}$are solutions of wave equations for the hydrogen atom $\mathrm{H}$ and the negatively charged ion $\mathrm{H}^{-}$, respectively, with modified Laplacian in which the radial part of the Jacobian (equal to $r^{2}$ in 3D space) is replaced by $J(r)=r^{2} \int f_{0}^{2}\left(\mathbf{r}^{\prime}\right) \delta\left(\left|\mathbf{r}^{\prime}\right|-r\right) d \mathbf{r}^{\prime}$. Functions $\Phi_{D^{0}}$ and $\Phi_{D^{-}}$and the energies $E\left(D^{o}\right)$ and $E\left(D^{-}\right)$, can be found by using any standard method from the atomic physics one of which is described in details in Ref. [4]. Particularly, for $\Phi_{D^{-}}$we use a three-parameter Hylleraas trial function [7].

\section{RESULTS AND DISCUSSION}

Below we present the results of calculation of the binding energies $E_{b}\left(D^{o}\right)$ and $E_{b}\left(D^{-}\right)$of the neutral and the negatively charged donors, defined as:

$$
\begin{aligned}
& E_{b}\left(D^{0}\right)=E\left(e^{-}\right)-E\left(D^{0}\right) ; \\
& E_{b}\left(D^{-}\right)=E\left(D^{0}\right)+E\left(e^{-}\right)-E\left(D^{-}\right)
\end{aligned}
$$

In Fig. 2 we show the dependencies of the ground state binding energies of the $\mathrm{D}^{-}$and the ratio of the binding energies of the $\mathrm{D}^{0}$ and $\mathrm{D}^{-}$located at the middle of the axis $\left(\xi_{\rho}=0, \xi_{z}=0.5 d_{0}\right)$ on the base radii of the disk, lens and conical pyramid with two different heights $d_{0}, 2 \mathrm{~nm}$ (solid lines) and $4 \mathrm{~nm}$ (dashed lines).

The binding energies presented in these figures should be compared with the corresponding values of the binding energies of the $\mathrm{D}^{-}$in 3D bulk, 0.055Ry* [7], in 2D bulk, $0.511 \mathrm{Ry}^{*}[9]$, respectively. As the QD radius is very large the structures can be considered as quasi-two dimensional systems with a strong but incomplete confinement in $\mathrm{z}$-direction due to the finite values of the thickness and the barrier height. Therefore as the radius increases and it becomes very large the binding energies of the $\mathrm{D}^{-}$tend to the values slightly lower than those for $2 \mathrm{D}$ bulk. In the region of the large base radii the largest binding energy presents in the conical pyramid and the disk presents the smallest binding energy. Comparing the curves for different structures one can see that effect of leaking of the wave functions in the barrier region due to decrease of the QD radius first occurs in conical pyramid following by lens and lastly in a disk. As result, the crossovers and the inversion of the binding energies order with the decrease of the base radius can be observed in Figs. 2. Besides, one can compare the maximum values of $\mathrm{D}^{-}$binding energies for different structures with corresponding values obtained previously for the SQD, 2.4Ry* and the cylindrical quantum well wire, $1.2 \mathrm{Ry}^{*}$ [4]. It is seen that the maximum values of the binding energies in quasi-two dimensional structures are lower than the corresponding values for the SQD.

In order to achieve a better understanding of the dimensional characteristics of the $\mathrm{D}^{-}$centers and the QD's geometrical-shape effect, we present in Fig. 2 the corresponding ratios $\sigma$ of $\mathrm{D}^{-}$to $\mathrm{D}^{0}$ binding energies as a function of the base radius. It has been shown in the paper [10] that values of the ratio $\sigma$ depend weakly on the potential shape and the limit values of $\sigma, 0.3,0.2,0.13$ and 0.05 can be considered as a typical for $0 \mathrm{D}, 1 \mathrm{D}, 2 \mathrm{D}$ and $3 \mathrm{D}$ heterostructures, respectively. One can see from Fig. 6 that the limit value oof OD 

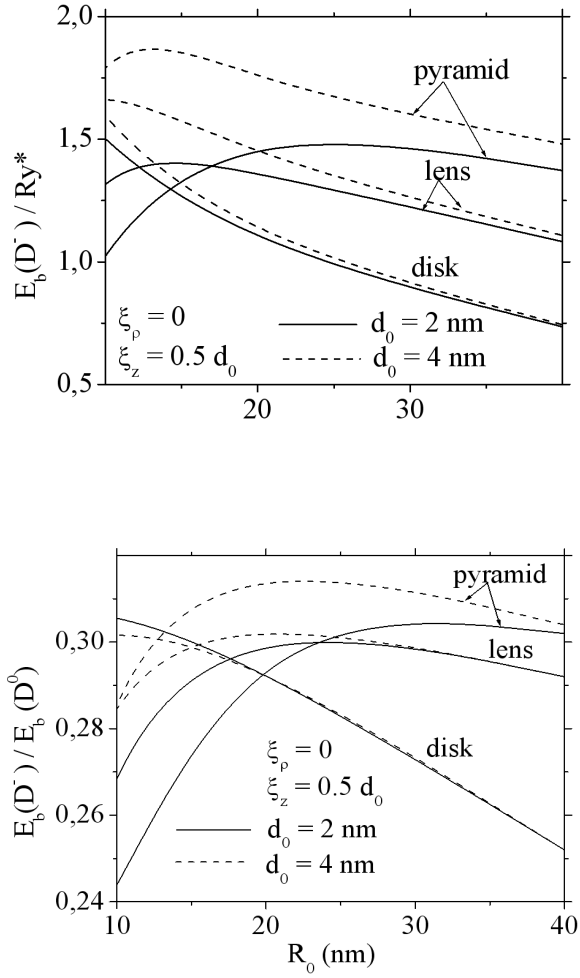

FIG. 2: Ground state binding energies $E_{b}\left(D^{-}\right)$and ratios $\sigma$ of $E_{b}\left(D^{-}\right)$to $E_{b}\left(D^{0}\right)$ as a function of the base radius $R_{0}$ for donors located at the middle of the axis of the disk, lens and conical pyramid with the heights $2 \mathrm{~nm}$ (solid lines) and $4 \mathrm{~nm}$ (dashed limes).

structures about 0.3 is also valid for all SAQDs independent of their geometrical shape. In the limit, as the radius becomes large, $\sigma$ decreases monotonically and it tends to its $2 \mathrm{D}$ bulk limit.

To study the effect of the donor position on the ground state binding energy we calculate the binding energies of the $\mathrm{D}^{-}$ centers located in different places of QDs. The results of calculation we show in the form of the curves in Fig. 3 and the contour plots in Fig.4. In Figs. 3 we have plotted the $\mathrm{D}^{-}$ binding energies and ratios $\sigma a s$ a function of $\xi_{p}$ for QDs with different shapes. The binding energies in all QDs decrease as the donor is removed from the axis. It is due to the fact that in these structures the motion of electron is mainly restricted close to axes independently on the donor position and therefore the electron-donor separation increases as the donor removes from the axis whereas the ratio $\sigma$ decreases from the value typical for $0 \mathrm{D}$ structure, about 0.3 to the value typical for 1D structure, about 0.2

The results of calculation for off-axis $\mathrm{D}^{0}$ and $\mathrm{D}^{-}$centers we show in Fig. 4 contour plots, which correspond to the level lines of the binding energies of the $\mathrm{D}^{-}$with different positions along a cross section in the middle of the pyramids, lens and disk perpendicular to the $y$ direction. The shadowed parts of figures indicate the cross section of the corresponding
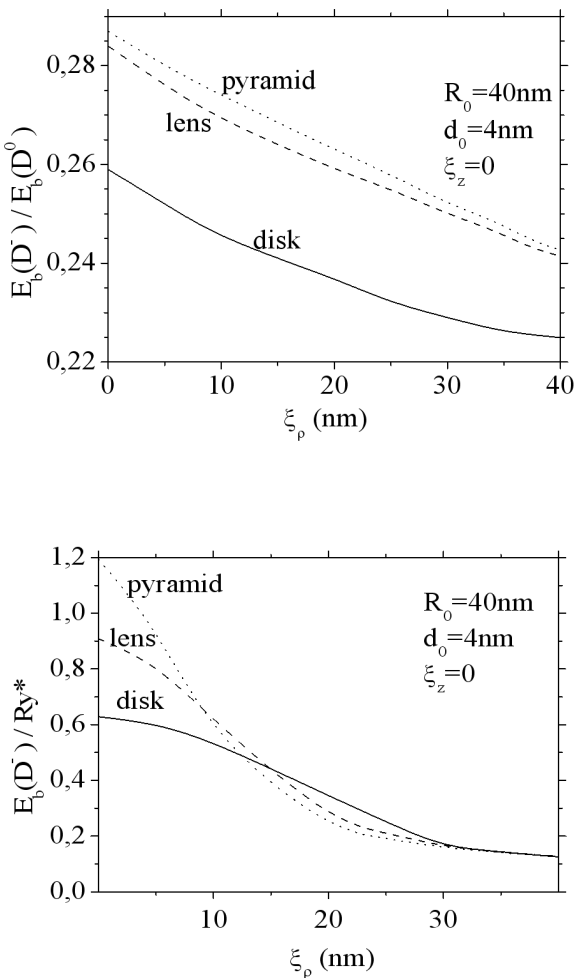

FIG. 3: Ground state binding energies of negative donors and ratios $\sigma$ as a function of the distance from the donor position to the axis in QDs with radius $40 \mathrm{~nm}$ and height $4 \mathrm{~nm}$.
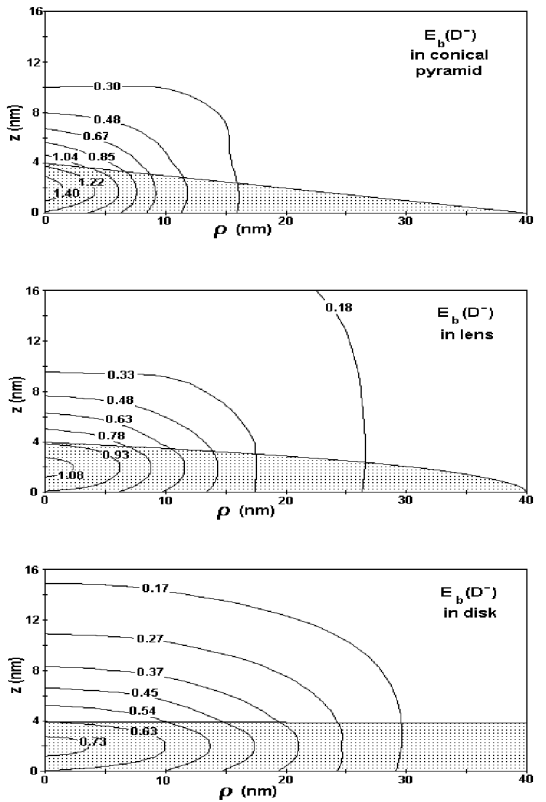

FIG. 4: Contour plots of binding energies of negatively charged donors corresponding to their different positions in a plane trough the axis of symmetry of the quantum dots with different profiles. 
structures. It is seen the evolution which undergoes the level lines as the shape of the QD varies from the conical pyramid to disk. Along the $\rho$ direction the level lines become more extended due to the decrease of the confinement as the QD's shape varies from the conical pyramid to disk.

In conclusion, we present the results of calculation the energies of the negatively charged donors and the ratios of the negative to neutral binding energies in flat SAQDs in order to study their dependencies on the dot shape, radius, height and the donor position.

\section{Acknowledgement}

This work was financed by the Colombian Agency COLCIENCIAS (Cod 1102-05-16923) and "Centro de Excelencia en Nuevos Materiales (CENM)" J. H. Marín wishes to thank the Universidad Nacional-Sede Medellín for the permission to study at the UIS.
[1] L. Jacak, P. Hawrylack, A. Wójs, In Quantum Dots (Springer, Berlin, 1997). A. Lorke, R. J. Luyken, A. O. Govorov, and J. P. Kotthaus, Phys. Rev. Lett. 84, 2223 (2000).

[2] G. Bastard, Phys. Rev. B 24, 4714 (1981).

[3] J. L. Zhu, J. H Zhao ,W. H. Duan, and B. L. Gu, Phys. Rev. B 46, 7546 (1992); J. L. Zhu, J. H Zhao, and J.-J. Xiong, Phys. Rev. B 50, 1832 (1994); J. L. Zhu, Xi Chen, Phys. Rev. B 50, 4497 (1994).

[4] I. D. Mikhailov, F. J. Betancur, R. Escorcia, and J. SierraOrtega, Phys. Stat. Sol. (b) 234, 590 (2002); I. D. Mikhailov, F. J. Betancur, R. Escorcia, and J. Sierra-Ortega, Phys. Rev. B 67, 156317 (2003)
[5] K. L. Janssens, F. M. Peeters, and V. A. Schweigert, Phys. Rev. B 63, 205311 (2001).

[6] F. J. Betancur, I. D. Mikhailov, and L. E. Oliveira, J. Phys. D: Appl. Phys. 31, 3391 (1998).

[7] H. A. Bethe and E. E. Salpeter, Quantum Mechanics of oneand two-Electron Atoms (Springer-Verlag, Berlin, 1957).

[8] J. L. Zhu, J. H. Zhao, W. H. Duan, and B. L. Gu, Phys. Rev. B 46, 7546 (1992).

[9] T. Pang and S. G. Louie, Phys. Rev. Lett. 65, 1635 (1990).

[10] I.D. Mikhailov, J. H. Marín, and F. García, Phys. Stat. Sol. (b) 242, 1636 (2005). 